\title{
Research of Piezoelectric Stack Pump and Hybrid Precision Stepper Drive Technology
}

\author{
QU Xing-tian ${ }^{a}$, YU Hai-yang ${ }^{b}$, Yang $\mathrm{Xu}^{\mathrm{c}^{*}}$ and Liu shuang ${ }^{\mathrm{d}}$
}

Dept. of Mechanical Manufacturing and Automation, Ji Lin University, Chang Chun 130025, China

aquxt@jlu.edu.cn, bhaiyang5146@sina.com, ${ }^{\mathrm{c} y a n g x @ j l u . e d u . c n,{ }^{d} 1146751689 @ q q . c o m}$

Keywords: Piezoelectric stack pump, Electro-rheological fluids, Precision driving, CFD

Abstract. The paper shows theoretical analysis and experimental study on the performance of the pump / valve hybrid drive system. Taking AE0505D16 type piezoelectric stack as power source, design and manufacture two new structures of pump: symmetric and staggered single-chambered dual piezoelectric stack pump. Comparing the two kinds of pump according to CFD analysis and experimental tests, we conclude that the staggered kind is a better structure. Testing the piezoelectric stack pump, discuss the impacts of voltage, frequency on the flow rate and output pressure. Through the experiment, measure the relationship between system driving force and the work performance of piezoelectric pump and electro-rheological valve, and the relationship between system step speed and piezoelectric pump's frequency and flow rate.

\section{Introduction}

The piezoelectric driving element can produce mechanical deformation under the action of the electric field [1,2]. Although the deformation is minimal, usually micron level, it can achieve nanometer resolution, while producing a large output force (up to hundreds of Newton), and can be high frequency changes, so it is very suitable for precision drive [3]. According to different working principle, the piezoelectric actuator can be divided into three categories: piezoelectric linear actuator, piezoelectric inertial actuator and piezoelectric stepping actuator. The three types of actuators are characterized by their characteristics and application areas [4]. Compared to the first two types of actuators, the piezoelectric stepping actuator can effectively alleviate the contradiction between large stroke and high resolution, and it has obvious advantages in multi degree of freedom precision drive with the requirements of large driving force, high resolution and large stroke $[5,6]$.

In recent years, with the development of electronic technology and material science, the research and exploitation of new piezoelectric actuator has achieved many significant results, and some of them have been produced as products to the market, which shows the bright future of the field [7]. The main application fields are as follows: MEMS and ultra-precision machining, biological engineering, medical science, integrated circuit manufacturing, optical fiber docking, micro operation and assembly, precision drive, etc. $[8,9]$.

In this paper, the structure of a linear precision actuator driven by piezoelectric stack is designed to achieve high precision, large driving force, fast response, large stroke; High performance of precision stepping drive is realized by the combination of piezoelectric and electro-rheological technology.

\section{Trial manufacture and experimental study of single-chambered dual piezoelectric stack pump}

\section{Structure and working principle of piezoelectric stack pump}

The piezoelectric stack pump structure is illustrated in Fig. 1. The globe valve is internal distributed cantilever valve, and the stack is NEC AE0505D16 which has a lesser quality and smaller size. Due to the small deformation of stack, only $12 \mu \mathrm{m}$, the pump is designed as single-chambered dual piezoelectric stack structure to increase the cavity volume change, while achieve the purpose of the large flow output. The pre tightening force of the PZT stack can be roughly adjusted by coarse 
adjustment pre-tightening nut and finely tuned by fine adjustment of nut with inclined block pre-tightening device.

The working principle of the PZT stack pump is that when the alternating voltage is applied to the PZT stack, the stack extends and shortens periodically, and the connected vibration diaphragm vibrates up and down, causing a change in the volume of the pump chamber. Then it causes the pressure difference between inside and outside of the pump chamber to make constant inflow and outflow of the fluid.

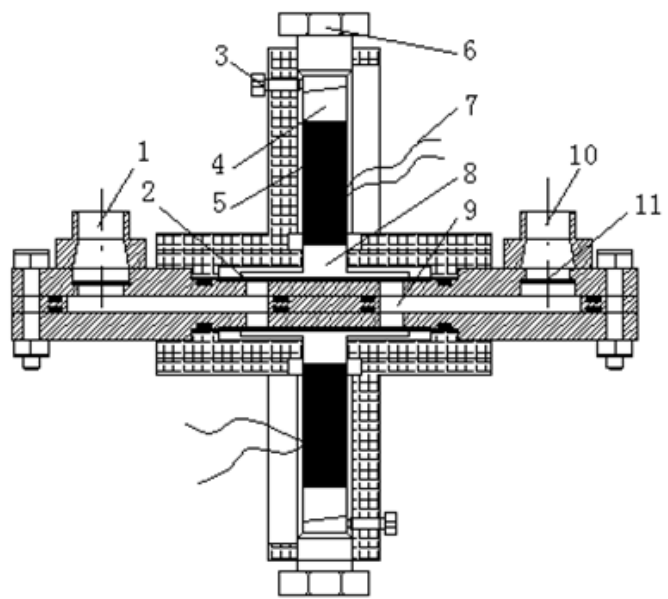

1. water inlet 2. vibration diaphragm 3. fine adjustment pre-tightening nut 4. inclined block pre-tightening device 5 . piezoelectric stack 6 . coarse adjustment pre-tightening nut 7. stack wire 8 . stack pedestal 9. fluid channel 10 . water outlet 11. valve block

Fig. 1 Structure of piezoelectric stack pump

\section{Design of the valve of piezoelectric stack pump}

The globe valve used in the pump in this paper is made of metal (e.g. beryllium bronze), as shown in Fig. 2 (a) (b), which belongs to cantilever beam valve. Fig. 2 (a) is the valve plate; Fig. 2 (b) is the valve base; Installation method is shown in Fig. 3: Valve plate (a) and base (b) are connected, and they are fixed on the support through the interference fit of the locating ring, achieving unidirectional cutoff effect. The material used for valve plate is beryllium bronze, the thickness is generally about $0.07 \sim 0.15 \mathrm{~mm}$. A key factor affecting the working performance of this kind of cantilever valve is the opening degree, and the flow cavity is proportional to the opening degree. In a certain frequency range, the opening degree of the valve plate increases with the increase of the frequency, and increases with the decrease of the thickness. This kind of cantilever beam valve is generally connected to the valve seat by viscose. This fixing method is inconvenient to disassemble, and it affects the whole service life of the pump to some extent; it can also use sealing ring press the valve plate into the cavity, but it will limit the height change of the cavity.

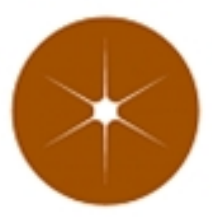

a)

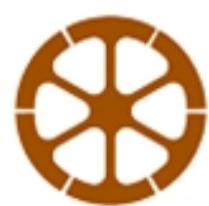

b)

Fig. 2 Cantilever beam valve style

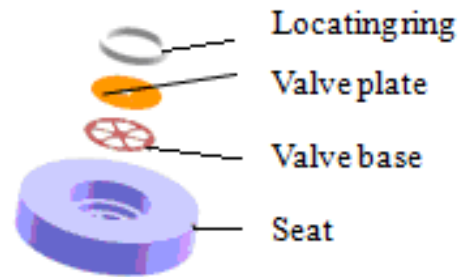

Fig. 3 Installation mode

\section{System vibration model of piezoelectric stack pump}

In order to maximize the volume change of the pump chamber, the piezoelectric stack pump should work under the system resonance frequency to obtain the maximum output flow. The system vibration characteristics related to the PZT stack pump are studied by means of vibration analysis of the system as followed. 
In Fig. $4, k_{1}$ is the equivalent stiffness of PZT stack; $k_{2}$ is the equivalent stiffness of seal diaphragm; $k_{3}$ is the equivalent stiffness of electro-rheological liquid; $c_{1}$ is the equivalent damping of seal diaphragm; $c_{2}$ is the equivalent damping of electro-rheological liquid; $m_{1}$ is the equivalent mass of piezoelectric stack plus seal diaphragm; $m_{2}$ is the equivalent mass of seal diaphragm plus liquid in the pump chamber. $y=F_{1} \sin (\omega t)$ is the equivalent driving force which the power supplies to the piezoelectric stack pump. When the PZT stack pump works, piezoelectric film, fluid and valve plate constitute a damped forced vibration system with three degrees of freedom.

System motion differential equation is as below:

$$
m+c x+k x=F(t)
$$

$k$ is the equivalent stiffness of the system and $k=k_{1}+k_{2}+k_{3} ; F(t)$ is excitation function of the system.

According to vibration analysis of the system, we conclude that:

Amplification factor:

$$
\beta=\frac{1}{\sqrt{\left(1-\lambda^{2}\right)^{2}+(2 \varsigma \lambda)^{2}}}
$$

$\lambda=1$ while system resonance, which means $\lambda=\omega / \omega_{n}=1$, at this time

$$
\beta=\frac{1}{2 \varsigma}=\frac{\sqrt{k m}}{c}
$$

According to Eq. (3), the amplification factor of the system under sinusoidal excitation is related to the equivalent stiffness, equivalent mass and equivalent damping of the system.

\section{Analysis of two different structures of single-chambered dual PZT stack pump}

In single-chambered dual PZT stack pump, the two PZT stack have two distribution modes: Staggered distribution, shown as Fig. 5 and symmetric distribution, shown as Fig. 6. Flow field analysis for the buffer cavity of the two distributions of PZT stack will be conducted by CFD.

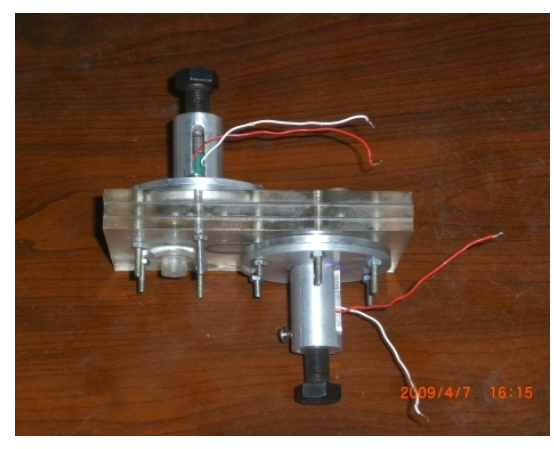

Fig. 5 Staggered distribution of PZT stack

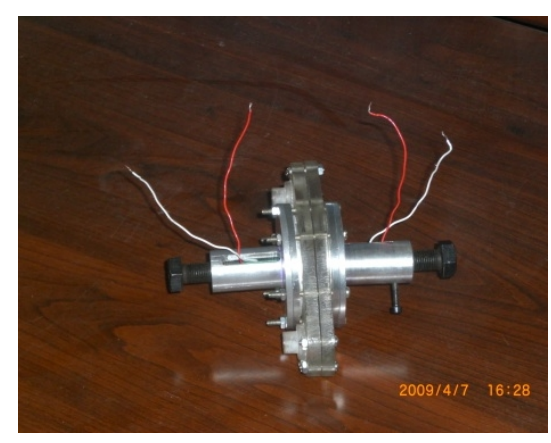

Fig. 6 Symmetric distribution of PZT stack

The two models are analyzed by using the same boundary conditions: the two valve inlets set a speed limitation with $0.1 \mathrm{~m} / \mathrm{s}$, the smooth surface, and the working medium is water. In the middle of the pump chamber, the vertical and horizontal cross sections are taken to display the velocity distribution, as shown in Figs. 7, 8, 9, 10. The speed change is more uniform in symmetric distribution PZT stack and the average output speed of staggered type is obviously larger than that of symmetric one.

At the inlet velocity of $0.1 \mathrm{~m} / \mathrm{s}$, the calculation shows that the exit velocity of staggered stack is $1.29 \mathrm{~m} / \mathrm{s}$, larger than the symmetrical distribution of $0.8 \mathrm{~m} / \mathrm{s}$. The outlet absolute pressure of the 
staggered distribution is slightly larger than that of the symmetric one, as shown in Figs. 11, 12, 101827.5Pa and 101340.6Pa, respectively. The energy output of the staggered distribution of the stack is greater by the Bernoulli Equation.

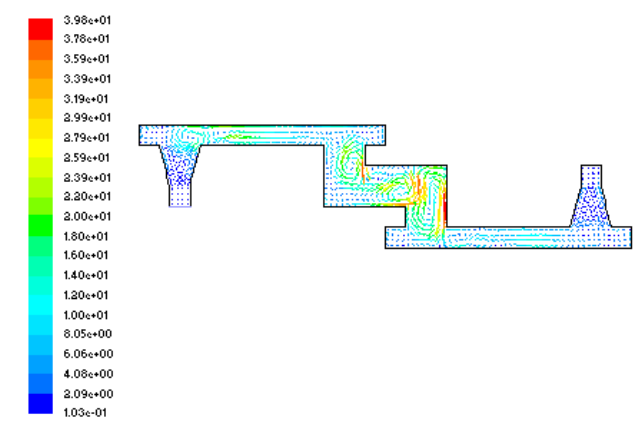

Fig. 7 Vertical surface flow velocity distribution of staggered structure

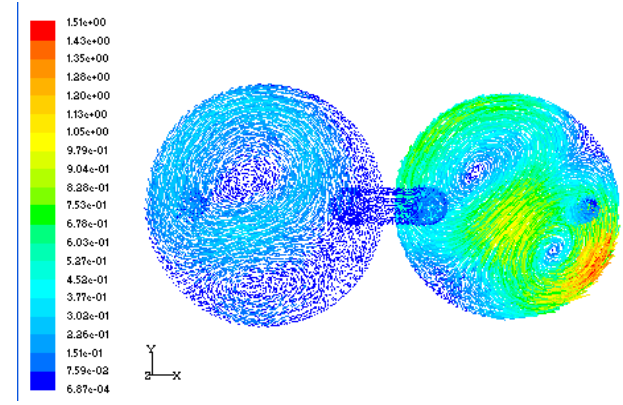

Fig. 9 Horizontal surface flow velocity distribution of staggered structure

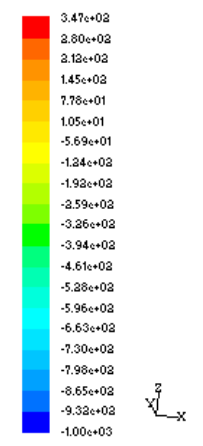

Fig. 11 Pressure distribution of staggered structure

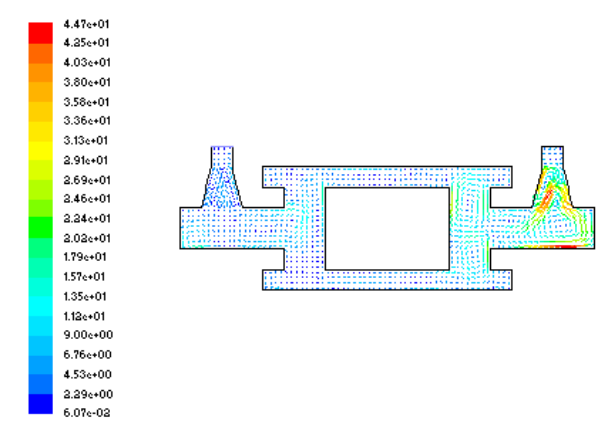

Fig. 8 Vertical surface flow velocity distribution of symmetric structure

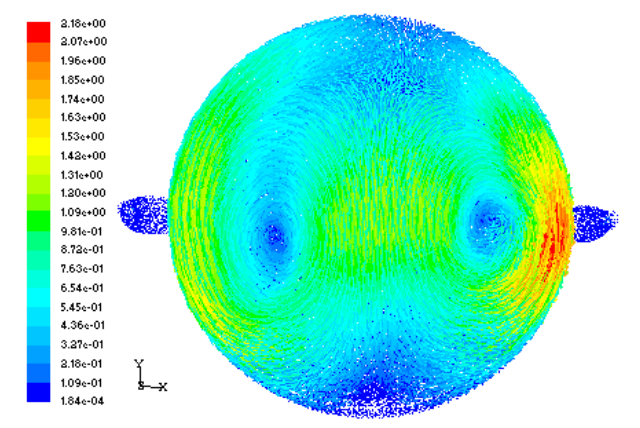

Fig. 10 Horizontal surface flow velocity distribution of symmetric structure

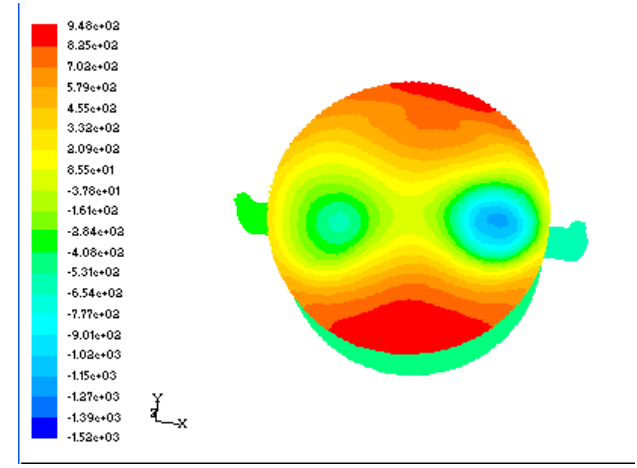

Fig. 12 Pressure distribution of symmetric structure

\section{Design of precision drive system for pump / valve}

The current piezoelectric stepping precision drive system, due to the mechanical connection between piezoelectric stack and the moving body, which affects the dynamic response of the system, its effective driving frequency is low, the step speed is slow, and continuous driving load step resolution is low. It is proposed that the high frequency and high precision fluid drive is realized by using the PZT stack pump without valve, and the high frequency switch control is realized by using the electro-rheological technology. The precision step is driven by precision hydraulic cylinder, which forms a precision pulse hydraulic stepping drive with high frequency, high step speed, high resolution, large stroke and large driving force. The system schematic diagram is as follows:

Fig. 13 is the principle diagram of PZT pump / ER hydraulic hybrid stepping drive system principle diagram. In this system, the PZT stack pump without valve is the power source. As a function of the 
switch control of the system, the electro-rheological valve is applied to applied electric field, making the ER liquid liquid phase and solid phase transition, playing a role in fluid closure. Precision hydraulic cylinder, as the terminal execution mechanism, is to achieve precision stepper driver.

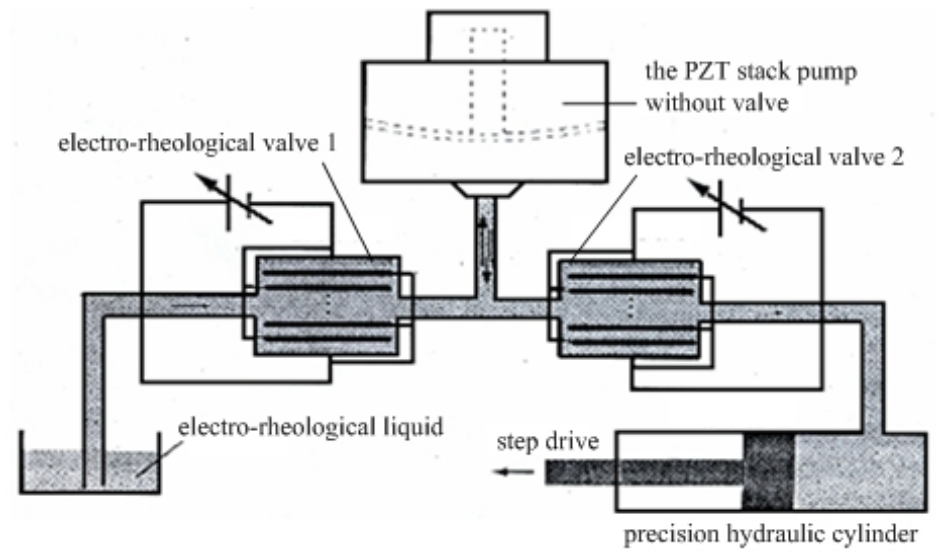

Fig. 13 the PZT pump / ER hydraulic hybrid stepping drive system principle diagram

The working mechanism of the system is as follows:

(1) In the initial state, the working unit is in a free state, and the electro-rheological liquid is filled with each cavity. Electrifying the ER valve 1, under the action of electric field, the ER liquid is changed from liquid phase to solid phase, making the valve in the close state. There is not electric field on valve 2 , so it is in a state of flow;

(2) Electrifying the PZT stack pump without valve, in a pulse voltage driven, the pump cavity volume becomes small, driving the ER liquid out of the pump cavity. As the ER valve 1 is closed, the liquid goes into the precision hydraulic cylinder through the valve 2, making the piston and the piston rod move a step in the direction of the icon under the liquid pressure;

(3) Electrifying the ER valve 2, under the action of electric field, the ER liquid is changed from liquid phase to solid phase, making the valve 2 in the close state;

(4) Cut off the electricity supply of the valve 1, and the ER liquid is changed from solid phase to liquid phase, making the valve 1 in the flow state;

(5) Cut off the power supply of the PZT stack pump, the PZT stack draws back, the cavity volume increases, due to the close state of valve 2, the ER liquid is sucked into pump cavity through valve 1;

(6) Electrifying the ER valve 1, under the action of electric field, the ER liquid is changed from liquid phase to solid phase, making the valve 1 in the close state. The valve 2 is in the close state, simultaneously;

(7) Cut off the power supply of the valve 2, and the ER liquid is changed from solid phase to liquid phase, making the valve in the flow state. At this point, the system returns to the state 1), completing a step cycle. Continuous repetition of the process, it can drive precision hydraulic cylinder piston stepping movement. Besides, the bridge connection for hydraulic system can realize forward and backward drive.

\section{Experimental study on the pump / valve hybrid precision drive system}

\section{Introduction of main experimental testing apparatus}

The main experimental apparatus used in this experiment are: HPV series piezoelectric ceramic drive power supply, as the power supply of the PZT stack pump, SP1641B function signal generator, multi-flow channel electro-rheological valve, DW - P502 - 5ACC6 high voltage DC power supply etc..

15 Performance test of the PZT stack pump 
(1) Effect of voltage and frequency on output flow

Fig. 14 is the curve of the impact of the input voltage on the output flow. It can be seen from the picture, the output flow rate increases with the increase of input voltage.

Fig. 15 shows the influential curve of the input frequency on the output flow. As can be seen from the picture, with the increase of the pump frequency, the output flow rate increases. When the frequency increases to about $25 \mathrm{~Hz}$, the output reaches maximum. After that, the output flow will gradually decrease with the frequency increase.

(2) Effect of voltage and frequency on output pressure

Fig. 16 is the influential curve of the input voltage on the output pressure at different frequencies. From the picture, we can see that with the increase of voltage, the output pressure gradually increases, and when the voltage increases to $100 \mathrm{~V}$, the pressure change trend becomes slow.

Fig. 17 is the influential curve of the input frequency on the output pressure under several voltages. The output pressure of the pump increases with the increase of the frequency. When the frequency comes about $7 \mathrm{~Hz}$, the output pressure reaches the maximum value of the experiment, and the water column is about $1000 \mathrm{~mm}$ (equivalent to $100 \mathrm{kPa}$ ). This shows the characteristics that the PZT stack pump is suitable for work under low frequency.

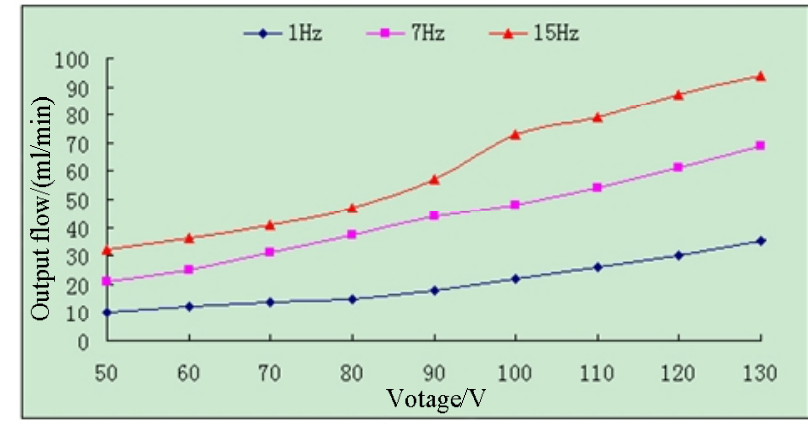

Fig. 14 Flow-voltage characteristic curve under different frequencies

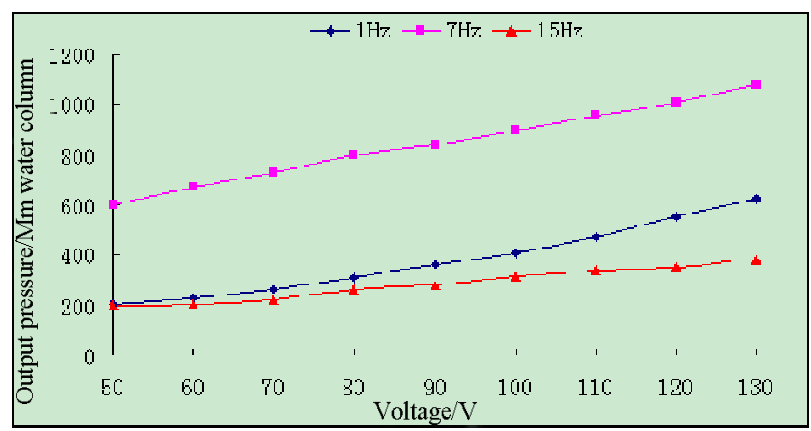

Fig. 16 Pressure-voltage characteristic curve under different frequencies

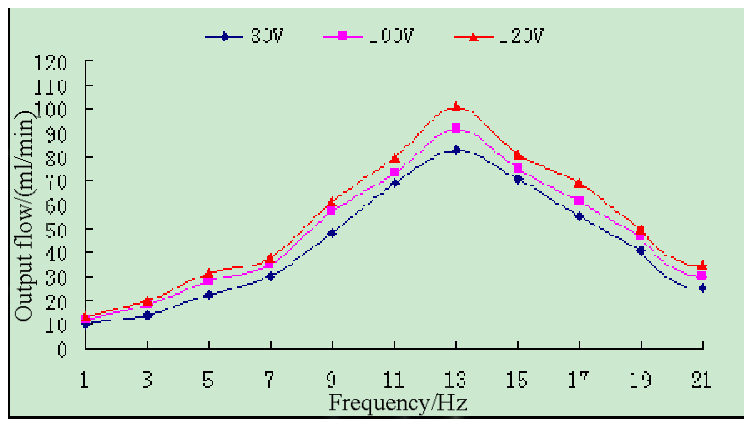

Fig. 15 Flow-frequency characteristic curve under different voltages

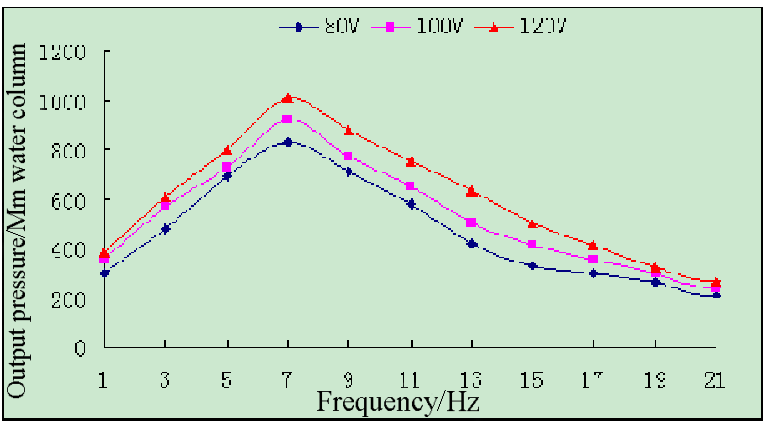

Fig. 17 Pressure-frequency characteristic curve under different voltages

\section{Performance test of precision drive system}

(1) Test of system driving force

Fig. 18 shows the relationship between the drive pressure and the drive force. In the graph, the driving force of precision actuator at $8 \mathrm{~V}$ voltage is about $0.28 \mathrm{Kgf}$. The driving force increases rapidly with the increase of the voltage. The static driving force reaches about $4 \mathrm{Kgf}$ when the voltage is $12 \mathrm{~V}$. The voltage continues to rise, and the driving force is maintained near the $5 \mathrm{Kgf}$. 


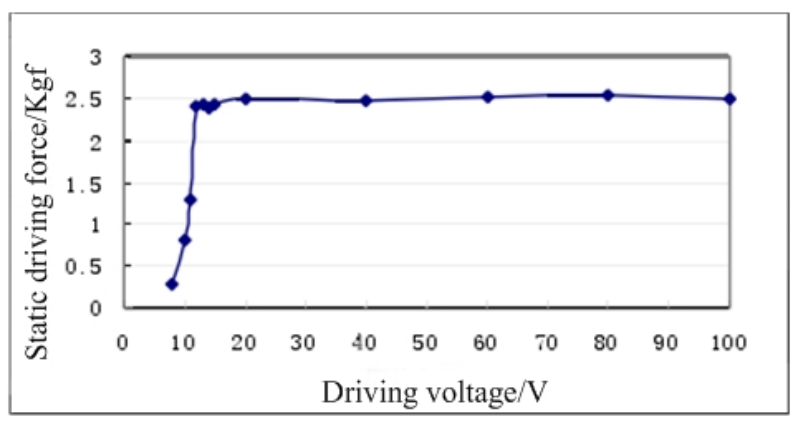

Fig. 18 Curve of the driving voltage and the driving force

\section{(2) System step test}

In this experiment, the step size of the driving mechanism under different driving voltage is tested. In fact, because of the interference of the external, the liquid reflux and compression in the pipeline, each step is not exactly equal to the other, so the average value of the 50 step is taken as the step size in the voltage. Fig. 19 shows the relationship between the step size of the precision drive actuator and the driving voltage in the condition of $1 \mathrm{~Hz}$.

\section{(3) System drive speed test}

In this paper, the maximum driving voltage of the precision actuator is $100 \mathrm{~V}$. As shown in Fig. 20, measure the driving speed of the precision actuator at different driving voltage under different frequencies of $1 \mathrm{~Hz}, 10 \mathrm{~Hz}, 5 \mathrm{~Hz}, 20 \mathrm{~Hz}$ and $40 \mathrm{~Hz}$. When the frequency is $40 \mathrm{~Hz}$ and the voltage is $100 \mathrm{~V}$, the precision actuator achieves the highest driving speed of $405 \mathrm{~m} / \mathrm{s}$. From the graph, it can be seen in a certain frequency, driving speed increases with the increase of the voltage. And when the voltage is constant, the driving speed increases with the increase of the frequency.

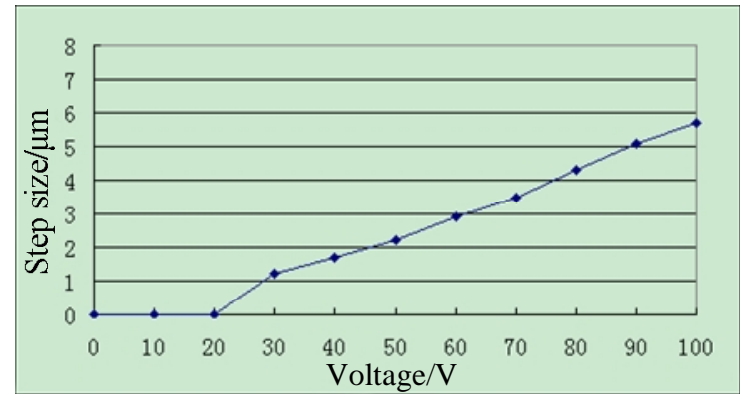

Fig. 19 Characteristic curve of the step size and the driving voltage

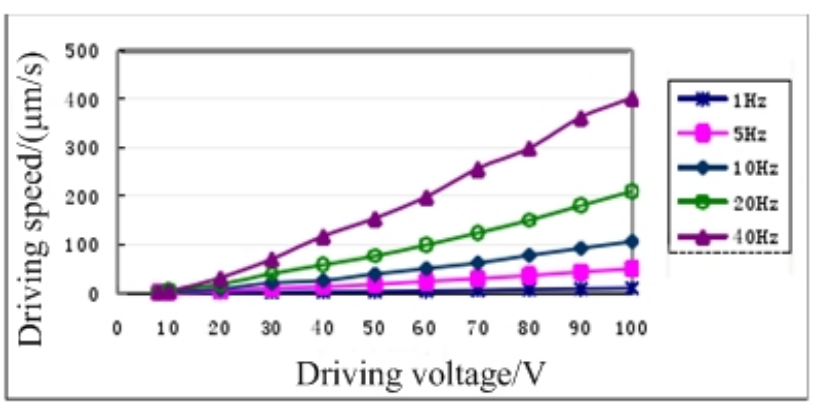

Fig. 20 Speed-voltage characteristic curve under different frequencies

\section{Summary}

Combining with the AE0505D16 type PZT stack produced by Japanese NEC-TOKIN used in this paper, a new type of stacked pump structure, single chamber dual piezoelectric stack pump is designed. This structure is a sealed cavity structure with a piston and metal diaphragm, which can improve the deformation area of the cavity and achieve a good sealing effect, and achieve the large output flow, the high output pressure. Based on this principle, prototypes of two kinds of pump are developed: symmetric and staggered single-chambered dual piezoelectric stack pump. Comparing the two kinds of pump according to CFD analysis and experimental tests, we conclude that the staggered kind is a better structure. Under the condition of water as the medium, the experimental platform of the piezoelectric stack pump is set up. The effects of voltage and frequency on the output flow and output pressure are studied by experiments.

Secondly, the precision, high frequency, high speed and stable step drive system is realized through the research of the hybrid precision drive system of the PZT stack pump / electro-rheological valve. 


\section{Acknowledgements}

The authors would like to be grateful to the Key Program of National Natural Science Foundation of China (Grant No. 51135006).

\section{References}

[1] GONG Feng fei. The Principle and Application of Microactuators. ELECTRONIC COMPONENTS \& MATERIALS, 2003, 6:32-35

[2] Oshida, Ryuichi, Okamoto, Yasuhiro , Higuchi, Toshiro. Development of smooth impact drive mechanism (SIDM) proposal of driving mechanism and basic performance Journal of the Japan Society for Precision Engineering. 1999. 65 (1): p.111-115

[3] ZHANG Hong-zhuang, ZENG Ping. Impact drive rotary precision actuator by piezoelectric bimorphs. Optics and Precision Engineering, 2005. 13(3.): p.298-303

[4] WUT, PAUL I R. Dynamic peak amplitude analysis and bonding layer effects of piezoelectric bimorph cantilevers. Smart Materials and Structure. 200143(1): p.203-210

[5] Choi S B, Han S S, Lee Y S. Fine motion control of a moving stage using a piezoactuator associated with a displacement amplifier. Institute of Physics Publishing. 2005,14(1): 222-230

[6] P.E. Tenzer, R.Ben Mrad. On amplification in inchworm precision positioners. Mechatronics, 2004, $14: 515-531$

[7] A. Sideman, S. Burns. Design and modeling of anelectrostrictive inchworm actuator. Mechatronics , 2004 , 14: 567-586

[8] Chanwoo Moon, Sungho Lee. A new fast inchworm type actuator with the robust I/Q heterodyne interferometer feedback. Mechatronics. 2006, 16:105-110

[9] WUT, PAUL I R. Dynamic peak amplitude analysis and bonding layer effects of piezoelectric bimorph cantilevers. Smart Materials and Structure. 200143(1): p.203-210 